\title{
Insulin resistance lasts 3 years after burn injury
}

Children with severe burn injuries experience stress-induced insulin resistance that lasts for years, new research has found. After injury “...a period of increased hypermetabolism, catabolism and marked inflammation accompanied by alterations in insulin sensitivity occurs that may persist for even longer than 3 years," says researcher Marc Jeschke of the University of Texas Medical Branch, Galveston, Texas.

Jeschke and coworkers previously showed that pediatric patients had significantly elevated levels of serum glucose and markedly increased insulin concentrations that correlated with the hypermetabolic state that occurs during the acute phase after a severe burn injury. This insulin-resistant, hyperglycemic state is a serious condition for patients with severe burns, as it increases the risk of infections and skin-graft loss, impairs wound healing and increases mortality. How long stress-induced insulin resistance persists after burn injury was not known, however, which prompted the researchers to conduct their prospective study.

The investigators used the oral glucose tolerance test to evaluate the insulin sensitivity of 194 children with burns that covered $>40 \%$ of their total body surface area and 95 nonburned, noninjured children acted as controls. Participants were monitored for up to 3 years after injury. In the children with severe burns, urinary cortisol, epinephrine and norepinephrine, certain serum cytokines and resting energy expenditure were increased for up to 3 years postburn. Levels of fasting glucose were significantly increased for 6 months, and levels of insulin and C-peptide were significantly elevated for the whole 3 -year period. Insulin-sensitivity indices also indicated that the injured children had peripheral and whole-body insulin resistance throughout the duration of the study.

These findings, says Jeschke, suggest that children with severe burns should be carefully monitored for a prolonged period of time for the development of type 2 diabetes mellitus and, in addition, indicate that these persistent metabolic disturbances could lead to growth delay. These findings stress the need for effective treatments for insulin resistance and hyperglycemia in children with severe burns. However, a number of barriers to effective treatment exist.

The benefit of tight glycemic control in pediatric intensive-care patients remains a contentious issue. Furthermore, children with severe burns are especially vulnerable to hypoglycemic episodes, because their enteral nutrition must be stopped periodically to enable changes to wound dressings and weekly operations. "We tend to recommend a less strict regime for the treatment of severely burned patients, that is, the maintenance of glucose levels $<7.8 \mathrm{mmol} / \mathrm{l}$," cautions Jeschke. Nevertheless, Jeschke's team aims to evaluate treatments for postburn, stress-induced insulin resistance in an upcoming clinical trial. The investigators are also examining the biochemical pathways involved in postburn insulin resistance, which are inadequately understood.

Carol Wilson

Original article Gauglitz, G. G. et al. Abnormal insulin sensitivity persists up to three years in pediatric patients post-burn. J. Clin. Endocrinol. Metab. doi:10.1210/ jc.2008-1947 